\title{
Bilateral Congenital Humeroradial Synostostis Presenting with Bilateral Proximal Radius Fractures: A Case Report
}

\author{
Elsiddig E. Mahmoud \\ Trauma \& Orthopaedics Department, Children's Health Ireland at Crumlin, Dublin, Ireland
}

\author{
Keywords \\ Congenital · Humeroradial synostosis $\cdot$ Radius fracture
}

\begin{abstract}
Congenital bilateral humeroradial synostosis (HRS) is a rare condition. It is generally divided into 2 categories. In the first group, which is mainly sporadic, additional upper limb hypoplasia typically coexists. In the second group, which is classically familial, HRS is commonly an isolated upper extremity anomaly. HRS can lead to variable degrees of functional disability. The clinical case reported here illustrates a possibly avoidable presentation of this uncommon condition. In this case report, we present a 6 -week-old male who presented with bilateral radius fractures. Radiography revealed congenital HRS at both elbows. No other associated congenital abnormalities were detected, and there was no family history of similar conditions in any first-degree relatives. In cases of congenital HRS, movement at the elbow joint is not possible. Parents who are unaware of this information might try to straighten their infant's elbows, which in turn may result in fractures of the proximal radius. Hence, early diagnosis and proper parental education could prevent fractures as a sequela of HRS.
\end{abstract}

(C) 2021 The Author(s).

Published by S. Karger AG, Basel

\section{Introduction}

Synostosis at the elbow is an uncommon condition that results from either skeletal malformations, soft tissue anomalies, or both [1]. The basis of this abnormality is the failure of separation and retention of the cartilaginous connection between bones around the elbow joint, which then undergoes endochondral ossification [2]. Despite this knowledge, the exact aetiology is unknown. Nevertheless, several genetic and environmental factors have been proposed to contribute to the development of this condition, including exposure to cocaine 
during pregnancy [3]. Synostosis at the elbow is subdivided by the type of bony fusion into humeroradial synostosis (HRS), which is the most common form, humeroradioulnar synostosis, and humeroulnar synostosis. HRS is further categorized into 2 main types: bone hypoplasia (class I) and joint maldevelopment (class II) [4]. In class I, which is mostly sporadic, the elbow is held in extension in about $75 \%$ of cases, and it is associated with ulnar ray hypoplasia. In contrast, in class II, which is nearly exclusively familial, the elbow is held in flexion in around 3 quarters of cases, and it is not associated with ulnar ray hypoplasia [1]. Class II HRS is frequently associated with Antley-Bixler syndrome, Pfeiffer syndrome, Apert syndrome, and multiple synostosis syndrome [5]. In this article, we report a rare case of isolated sporadic class II bilateral HRS presenting with bilateral proximal radius fractures.

\section{Case Report/Case Presentation}

We present the case of a 45-day-old Sudanese male infant whose parents were of low socioeconomic background. The presenting complaint was crying with any attempt to move his elbows that had been ongoing for about 2 weeks. They also reported that he was keeping both elbows in a flexed position since birth for which they were encouraged to do stretching exercises by their relatives. His parents were not related, they had no history of musculoskeletal malformation, and he was their first child. His mother gave no history suggesting exposure to teratogenic agents or maternal infection during pregnancy. He was born at full term with a normal vaginal delivery, and, although his mother reported that he had delayed crying after birth, he was not admitted to the neonatal intensive care unit. Physical examination revealed proximal forearm tenderness bilaterally, and both elbow joints were stiff with 85 degrees of flexion and neutral forearm rotation. Apart from slightly increased muscle tone in the lower extremities, the rest of his examination was normal. In particular, there were no findings of other joint involvement or other skeletal anomalies. Similarly, no abnormalities were detected upon physical examination of his chest, abdomen, and genitalia. Nonaccidental injury was excluded after obtaining a detailed history, performing a meticulous physical examination, conducting the relevant investigations, and involving a social worker. Plain radiographs showed bilateral HRS with bilateral proximal radius fractures in different stages of healing (Fig. 1). No associated congenital abnormalities were detected on echocardiography and urinary system ultrasound. The fractures were treated non-operatively by advising his parents not to manipulate the elbows. No casts or splits were applied. The patient was referred to a paediatrician, highlighting the history of delayed crying after birth in addition to the mildly increased muscle tone in the lower extremities. The patient was seen 6 weeks later, and no tenderness was detected over the proximal radius on both elbows. X-rays revealed fully healed fractures (Fig. 2).

\section{Discussion/Conclusion}

Congenital fusion between the humerus and radius is due to altered development of the bones of the upper extremity between weeks 4 and 10 of embryonic life [4]. The majority of reported cases of bilateral HRS are familial [6]. Sporadic cases are commonly accompanied by significant underdevelopment of the ulna and hand [6]. We report an atypical case of bilateral HRS with no family history or coexisting limb anomalies.

Hunter and Rudd reported a case of HRS with an associated proximal radius fracture, and they stated that the fracture was most likely due to birth injury [6]. This is a possible explanation in our case. However, the history of pain in this patient did not begin at birth but only

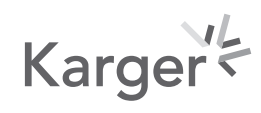


Fig. 1. Elbow radiographs showing humeroradial synosteses and proximal radii fractures in different stages of healing. a Right elbow. b Left elbow.
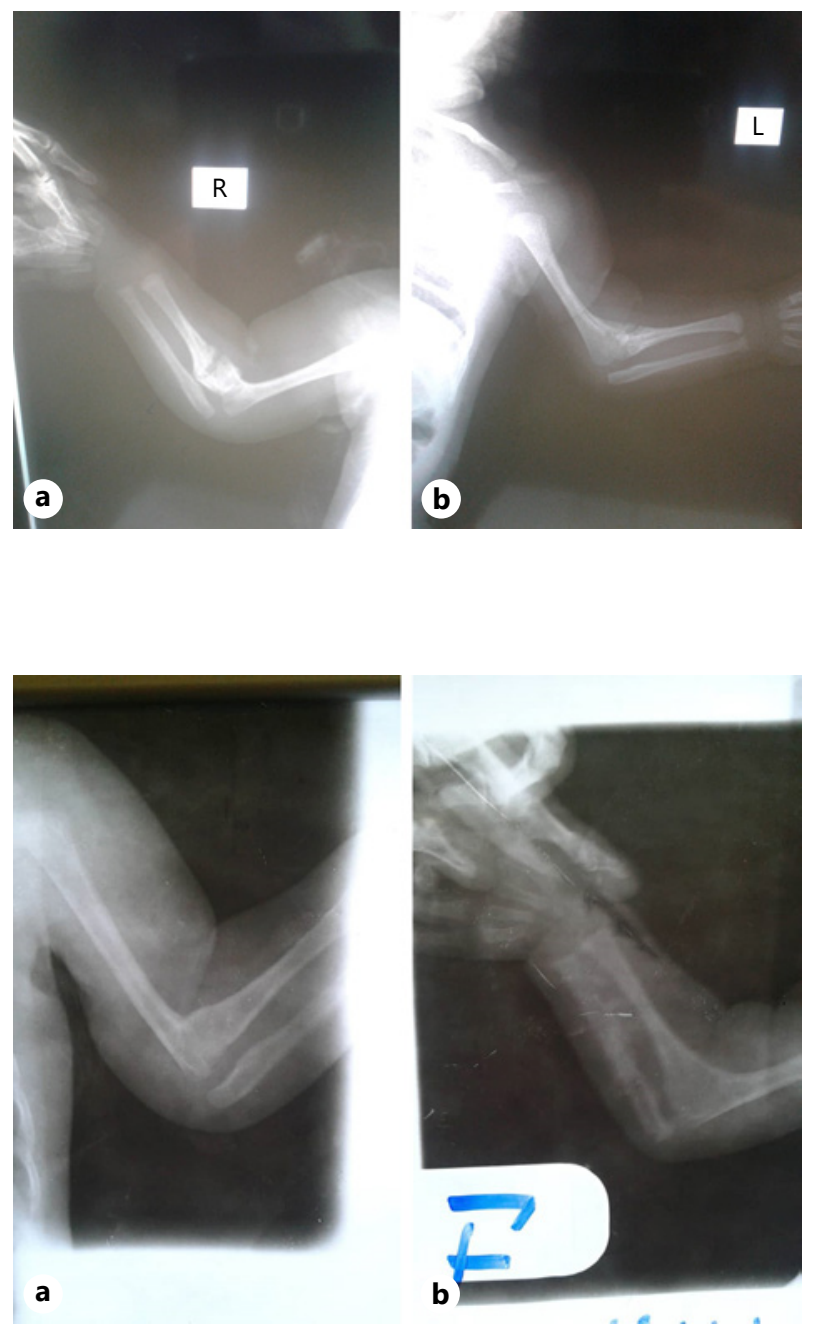

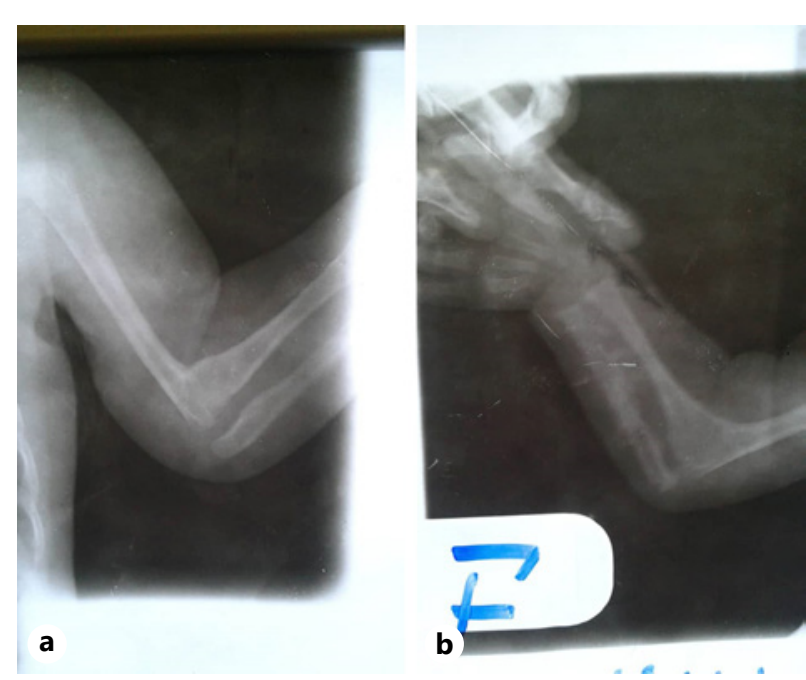

a
Fig. 2. Elbow radiographs 6 weeks later showing healed fractures. a Right elbow. b Left elbow.

developed about 2 weeks before presentation. The parents reported that they attempted to straighten their child's elbows repeatedly based on the advice of their relatives. Therefore, we believe that manipulation performed by the parents could have been the cause of the fractures. We propose that early diagnosis and education of family members may prevent the incidence of radius fractures in HRS patients, especially when dealing with families of low education level.

Most HRS patients do well with non-operative management, particularly if the elbow is fixed in a functional position [7]. The value of surgical intervention in HRS is uncertain. Although success in the form of good post-operative elbow range of motion has been reported [8], there are significant concerns in the literature about possible recurrence and need for reoperation $[6,7]$. Operative treatment is not recommended when dealing with congenital fusion around the elbow except in bilateral involvement with functional disabilities [9].

Different surgical techniques have been reported to promote patient motor function. Sahdi et al. utilized a modified French osteotomy (from $30^{\circ}$ to $90^{\circ}$ of elbow flexion) in a fouryear-old boy with bilateral HRS [10]. Kakarala et al. [11] used distraction osteogenesis (from $70^{\circ}$ to $110^{\circ}$ of elbow flexion) in a 13-year-old patient with bilateral congenital humeroradioulnar synostosis. Both studies reported improved functional outcomes post-operatively. 
We chose to adopt the conservative treatment option recommended by Nema et al. [12] in treating this child because majority of these patients do well, especially if it is an isolated anomaly. Furthermore, he is too young for surgical intervention as most of the reported surgical interventions were performed on older children $[10,11]$. Moreover, there is no solid evidence of the superiority of operative over conservative treatments for this condition. Nevertheless, if significant functional limitation is detected in future follow-up appointments, surgical intervention will be considered.

HRS in both elbows is an uncommon condition that can present with proximal radius fractures. Early diagnosis and parental counselling, specifically for those with low educational levels, could prevent the occurrence of fractures in these patients.

\section{Statement of Ethics}

Written informed consent was obtained from the patient's father for publication of this case report and accompanying images.

\section{Conflict of Interest Statement}

The author declares no explicit and potential conflicts of interest associated with the publication of this article.

\section{Funding Sources}

The author received no funding relevant to this study.

\section{References}

1 Swenson V, Spinek A. A rare case of congenital humeroradioulnar synostosis from medieval Pawłów Trzebnicki, Poland. Int J Osteoarchaeol. 2020 Mar/Apr;30(2):256-63.

2 Bhatt CR, Mehta CD. Case report: congenital radioulnar synostosis and its embryological correlation and functional assessment. J Anat Soc India. 2011 Dec;60(2):236-8.

3 Marles S, Reed M, Evans J. Humeroradial synostosis, ulnar aplasia and oligodactyly, with contralateral amelia, in a child with prenatal cocaine exposure. Am J Med Genet. 2002;116A(1):85-9.

4 McIntyre JD, Benson MK. An aetiological classification for developmental synostoses at the elbow. J Pediatr Orthop B. 2002 Oct;11(4):313-9.

5 McIntyre JD, Brooks A, Benson MK. Humeroradial synostosis and the multiple synostosis syndrome: case report. J Pediatr Orthop B. 2003 May;12(3):192-6.

6 Hunter AGW, Cox DW, Rudd NL. The genetics of and associated clinical findings in humero-radial synostosis. Clin Genet. 1976 May;9(5):470-8.

7 Jacobsen ST, Crawford AH. Humeroradial synostosis. J Pediatr Orthop. 1983 Feb;3(1):96-8.

8 Mnaymneh WA. Congenital radio-humeral synostosis. A case report. Clin Orthop Relat Res. 1978 Mar-Apr; (131):183-4.

9 Mostert AK, Tulp JA. Congenital synostosis of the proximal forearm. Orthop Trauma. 2002 Oct;16(5):395-7.

10 Sahdi H, Rasit AH, Khoo CS, Bojeng A, Nur-Alyana BA. Modified French Osteotomy for humeroradial synostosis in a child with multiple synostoses syndrome: a case report. Malays Orthop J. 2019 Jul;13(2):52-5.

11 Kakarala G, Kavarthapu V, Lahoti O. Distraction osteogenesis to improve limb function in congenital bilateral humeroradioulnar synostosis. Acta Orthopaedica Belgica. 2007 Jan;72(6):765-8.

12 Nema S, Vyas GS, Sirsikar A, Bhoj PK. Congenital humeroradial synostosis: a case report. Malays Othop J. 2012 Jun;6(Suppl A):41-2.

\section{Karger'₹}

